\title{
Pediatric Continuous Brachial Plexus Catheter for a Case of Complex Regional Pain Syndrome (CRPS)
}

\author{
Reda Tolba' ${ }^{1}$, Rhamee Badr ${ }^{2}$, Maged Guirguis', Loran Mounir Soliman ${ }^{3}$ \\ ${ }^{1}$ Anesthesiology and Pain Management Department, Ochsner Health System, New Orleans, USA \\ ${ }^{2}$ Tulane University School of Medicine, New Orleans, USA \\ ${ }^{3}$ Anesthesiology Institute, Cleveland Clinic Foundation, Cleveland, USA \\ Email: redatolba@hotmail.com
}

Received 24 November 2014; revised 25 December 2014; accepted 7 January 2015

Copyright (C) 2015 by authors and Scientific Research Publishing Inc.

This work is licensed under the Creative Commons Attribution International License (CC BY). http://creativecommons.org/licenses/by/4.0/

(c) (i) Open Access

\begin{abstract}
Introduction: Complex regional pain syndrome (CRPS) is a disorder characterized by pain as well as a myriad of sensory, autonomic, and motor disturbances. We are reporting a case of child diagnosed with CRPS and successfully treated with supraclavicular brachial plexus catheter infusion of local anesthetic. Case Report: An eight-year-old male underwent a left thoracotomy, repair of esophageal vascular ring, and translocation of the left subclavian to the left carotid artery. Post-operative course was relevant for severe intractable left shoulder and left arm pain associated with allodynia and hyperalgesia. A supraclavicular catheter was inserted, and an infusion of ropivacaine $0.2 \%$ was started. The child was sent for physical therapy as he gradually regained all functions of his left arm and resumed his regular activities. Conclusion: Continuous supraclavicular brachial plexus catheter infusion of local anesthetic is a valuable method of reducing pain in severe cases of upper extremity pediatric CRPS and may be safer and more effective than other invasive measures such as sympathetic blocks and epidural catheterization. Further research surrounding the diagnosis and treatment of pediatric CRPS is needed to allow early diagnosis and treatment and to improve outcome.
\end{abstract}

\section{Keywords}

Complex Regional Pain Syndrome (CRPS), Supraclavicular Brachial Plexus Catheter, Pediatric

\section{Introduction}

Complex regional pain syndrome (CRPS) is a disorder characterized by pain, as well as a myriad of sensory, 
autonomic, and motor disturbances, and represents a significant medical entity [1] [2]. CRPS includes previous pain conditions with vasomotor and sudomotor dysfunction, including causalgia, Sudeck's atrophy, neuroalgodystrophy, shoulder-hand syndrome and reflex sympathetic dystrophy (RSD). CRPS can be subdivided into two categories, CRPS I and CRP II. CRPS I, formerly known as RSD, does not involve definable major nerve injury while CRPS II, formerly known as causalgia [3], involves definable major nerve injury. The diagnosis of CRPS is clinical, based on the findings in history and physical examination. Pain with allodynia and signs of autonomic instability in accordance with the Budapest diagnostic criteria are required to make the diagnosis. Pain should be disproportionate to the inciting event, if any. Radiological studies, such as bone scan and MRI, are important to rule out underlying orthopedic anomalies that may explain symptomatology, and if present, would exclude the diagnosis of CRPS [3]-[7]. There are no laboratory tests used to confirm the diagnosis of CRPS. CRPS is primarily a clinical diagnosis; however, functional imaging, visual analog scales, and devices to quantify temperature and mechanical allodynia are available to corroborate physical exam findings [3].

Management of CPRS in children is multidisciplinary and should aim to provide adequate pain control and appropriate physical therapy. Conservative treatment modalities include physical therapy, behavioral therapy and management with opioids and membrane stabilizers. More invasive approaches such as transcutaneous electrical nerve stimulation (TENS), sympathetic blocks, tunneled epidural catheters, regional nerve blocks, as well as more aggressive surgical approaches may be necessary in recalcitrant cases. In this case we report the use of supraclavicular brachial plexus catheter continuous infusion for the treatment of upper extremity CRPS in an 8-year-old child. This case study details how continuous supraclavicular brachial plexus catheter infusion of local anesthetic for 7 days helped control the child's pain and allowed for the initiation and maintenance of physical therapy with the eventual restoration of the patient's normal activity level.

\section{Case Report}

Eight-year-old male underwent a left thoracotomy, repair of esophageal vascular ring, and translocation of the left Subclavian to the left carotid artery. The child sustained a stable intra-operative course. Post-operatively, the patient complained of continuous burning 10/10 pain over his left shoulder with marked limitation of arm movement. The pain progressed to involve the whole left arm with associated allodynia and hyperalgesia on exam. The pain could not be controlled with oral pain medications, membrane stabilizers and IV opioids. X-rays and MRI of the shoulder failed to reveal any pathology of the brachial plexus or the shoulder itself and subsequently the diagnosis of CRPs was made. This was based on clinical finding of allodynia and hyperlagesia, and the presence of severe pain out of proportion that cannot be explained by radiological and EMG findings. The decision was made to perform a left supraclavicular brachial plexus block and catheter insertion for continuous infusion of local anesthetic. Under general anesthesia, using ultrasound and nerve stimulator guidance, a supraclavicular catheter was inserted. An initial bolus of 12 cc mepivacaine $1.5 \%$ was injected with adequate spread around the nerve. The catheter was then tunneled and secured. The patient experienced dramatic pain relief after the block, however the next day, the catheter dislodged. At this time, the decision was made to place a tunneled cervical epidural catheter under general anesthesia using fluoroscopy guidance. This procedure was ineffective at controlling the pain. The patient continued to experience significant burning pain in his left arm and shoulder. On account of the significant pain reduction experienced after the initial supraclavicular block, another supraclavicular brachial plexus catheter was inserted under ultrasound guidance. Ropivacaine $0.2 \%$ infusion was used with 5 cc basal rate, 3 cc bolus, and 60 minutes lockout intervals. The patient was sent to rehabilitation with the catheter still in place. The catheter was kept in place for seven days with considerable pain relief as the patient started his rehabilitation program.

The child was followed later in surgery clinic after finishing successful rehabilitation program. He gradually regained all functions of his left arm and resumed his regular activities.

\section{Discussion}

The occurrence of CRPS in children was questioned for a long time however several studies and case reports have since come to describe the syndrome and lend more credence to its existence as a clinical entity. Unlike adults, involved children are generally female and develop CRPS following minor trauma. CRPS in the pediatric population, in contrast to adults, mainly involves the lower extremity, especially the foot [8] [9]. Additionally, many investigators found psychological issues to be prevalent among this patient population although newer 
findings suggest otherwise [10]-[12]. Some of these differences between adult and pediatric cases of CRPS as well as data from early case reports in the 1970s detailing spontaneous resolution in pediatric patients [13]-[15] have led to a divergence in the scientific/clinical community. There is a contingent that believes that pediatric CRPS should be treated differently from adult CRPS, and for the most part more conservatively [16]. These sentiments as well as an overall lack of literature on pediatric CRPS may possibly underlie the delay in diagnosis of this condition as well as high reoccurrence rates. Lowe et al. describes a mean time to diagnosis of 13.6 weeks and a reoccurrence rate of $20 \%$ in his patient population [17]. The notion that pediatric CRPS is a milder clinical entity than its adult counterpart as well as the push for solely conservative treatment paradigms is dangerous.

Conservative management via physical therapy and psychotherapy alone (and possibly oral analgesia) may suffice for a majority of patients; however this will not be curative in all cases. Low describes a study of 20 children diagnosed with CRPS at a major children's hospital over a 4-year period. Although a large percentage of them had resolution of symptoms following intense physiotherapy and psychotherapy, almost $70 \%$ of these patients required adjuvant medication to enable them to participate in physiotherapy, $40 \%$ required in patient hospital treatment, and $20 \%$ had a relapse episode [8]. Furthermore, this $20 \%$ may be underrepresented as patients may have followed up at another hospital/facility or may yet still follow up sometime in the future. Wilder et al. found that 38/70 of the pediatric CRPS patients in their case series continued to have some residual pain and dysfunction despite conservative treatment with physical therapy, transcutaneous electronic nerve stimulation, psychological therapy, and TCAD and even despite more invasive sympathetic blocks in several of these patients [15]. Other studies demonstrate similar findings, documenting cases of recalcitrant pediatric CRPS and the inefficacy of conservative measures to resolve symptoms [4] [17] [18]. A 2009 study using a mailed questionnaire found that at least half of young adults who experienced CRPS I during childhood an average of 12 years prior to the study, still report spontaneous pain (52\%), pain after exercise (57\%) and other sensory, motor, or autonomic symptoms [19]. Hence, pediatric CRPS is not a mild clinical entity and in many cases is very resistant to conservative treatment.

A recent study found that pain induced persistent reorganization and hyper-connectivity of the cortical, limbic, and basal ganglia pain circuits in pediatric CRPS patients even after treatment and symptom resolution [20]. This "hyper-connectivity" may underlie CRPS reoccurrence and one may speculate that the higher reoccurrence rates of CRPS in children, as high as 30\% - 50\% in some studies [5] [12] [21], may be due to delays in diagnosis and possible insufficient treatment which allow time for such structural changes to take place. The swift elimination of the pain in pediatric CRPS may preclude the development of such structural changes. Further research is necessary to formally establish a link between persistent structural changes and CRPS persistence and reoccurrence.

The prognosis and responses to the same therapy vary among children suffering from CRPS [22]. Treatment modalities utilized in the past include physical therapy, behavioral therapy, antidepressants, TENS, sympathetic blocks, peripheral nerve blocks, spinal cord stimulation and sympathectomy [15]. A multidisciplinary approach utilizing analgesia, physical therapy, and behavioral therapy is ideal, but physical therapy plays the predominant role in treatment of CRPS. Sherry et al. demonstrated in her prospective follow up study of 103 children with CRPS treated with aggressive physical therapy alone that $92 \%$ of the children were symptom free immediately following therapy, and of those children followed for more than 2 years, $88 \%$ of them were symptom free, although some did have reoccurrences in between [5]. As shown in this case, patients may not be able to tolerate physical therapy and may require significant pain control. Pain control should start with more conservative oral analgesia and escalate to more invasive procedures. Invasive procedures such as peripheral nerve blocks, epidural anesthesia, and sympathetic blocks are not performed to treat CRPS as much as they are aimed at providing adequate pain control so that the patient can swiftly and effectively engage in physical therapy [15].

Sympathetic blocks, commonly stellate ganglion blocks for upper extremity pathology or lumbar sympathetic blocks for lower extremity pathology, are often used in refractory cases of CRPS in adults, and some clinicians have utilized these in recalcitrant pediatric cases as well [15] [21]. A role for the sympathetic nervous system in the induction and maintenance of pain in CRPS arises from the observation that CRPS patients undergo pain relief following sympathetic blockade. These patients were subsequently said to have sympathetic maintained pain [12]. Sympatho-afferent coupling as well as sympathetic induced vasoconstriction, ischemia and subsequent acidosis of affected tissue in CRPS patients may possibly underlie these findings [12] [23]-[25], however randomized control trials have not been able to show that sympathetic blocks are any more effective than placebos [26]. As the incidence of placebo effects is high in CRPS [27], it is possible that sympathetic mechanisms may 
in reality have little bearing on pain in CRPS. Microneurography in 24 patients with CRPS I and II found no evidence of nociceptor activation after sympathetic provocation maneuvers [28], further validating this notion that sympathetic hyperactivity is not responsible for pain in CRPS and that sympathetic blocks may not carry as much utility as once thought in this condition. Furthermore, the use of stellate/lumbar sympathetic blocks in children poses safety concerns due to the potential for neuraxial trauma as well as the inherent risk associated with the sedation or general anesthesia required for these interventions [29]. As these blocks are often multiple in nature and spaced across several days or weeks, the cumulative risk associated with them is considerable. Additionally, parents may also have to take significant time off of work to bring their children back and forth from their homes to the hospital for these procedures, and hospitals may incur increased costs associated with multiple OR visits [29].

Continuous brachial plexus supraclavicular nerve catheter placement may be a great, cost effective alternative for pain management in recalcitrant upper extremity CRPS. A retrospective study of 25 patients, 17 of whom were diagnosed with CRPS, showed significant improvement in pain and ROM following Bupivacaine brachial plexus injections [30]. While a continuous epidural catheter may also be effective, it failed to cause any pain relief in this case. Continuous epidural catheterization for pain control, unlike continuous supraclavicular catheterization, does not allow for unilateral blockade to the extremity and also assumes the risk of neuraxial trauma [29]. Supraclavicular catheters are fairly safe and the most common complication associated with these procedures is a superficial skin infection at the skin insertion site [31], although tunneling a continuous catheter seems beneficial in reducing risk of infection by decreasing clinically relevant movements of the catheters [32]. Epidural abscesses and infections, while only rare complications of epidural catheterization, have the potential to be much more devastating just given their location and even without direct trauma, abscess formation, or hematoma, cases of ischemic injury to the spinal cord have been documented in children undergoing epidural anasthesisa [33].

Certainly, there is a need for further research surrounding the diagnosis and treatment of pediatric CRPS. Delays in diagnosis, higher reoccurrence rates in pediatric cases of CRPS than in adults, instances of conservative measure failure, as well as possible biological mechanisms linking inadequate pain control and reoccurrence, all highlight the need for change. It follows that better diagnostic criteria for pediatric CRPS must be developed and that a more comprehensive and at times invasive approach to treating pediatric CRPS may be necessary. This case study illustrates that pediatric CRPS is not a mild clinical entity and that invasive measures may be useful to expedite physical therapy and recovery.

\section{Conclusion}

Continuous supraclavicular brachial plexus catheter infusion of local anesthetic is a valuable method of reducing pain in severe cases of upper extremity pediatric CRPS and may be safer and more effective than other invasive measures, such as sympathetic blocks and epidural catheterization. Further research surrounding the diagnosis and treatment of pediatric CRPS is needed to allow early diagnosis and treatment and to improve outcome.

\section{References}

[1] Bogduk, N. and Merskey, H. (1994) Classification of Chronic Pain: Descriptions of Chronic Pain Syndromes and Definitions of Pain Terms. Second Edition, IASP Press, Seattle, 212.

[2] Stanton-Hicks, M., Baron, R. and Boas, R. (1998) Complex Regional Pain Syndromes: Guidelines for Therapy. Clinical Journal of Pain, 14, 155-166. http://dx.doi.org/10.1097/00002508-199806000-00012

[3] Freedman, M., Greis, A.C. and Marino, L. (2014) Complex Regional Pain Syndrome. Physical Medicine and Rehabilitation Clinics of North America, 25, 291-303. http://dx.doi.org/10.1016/j.pmr.2014.01.003

[4] Stanton, R.P., Malcolm, J.R., Wesdock, K.A., et al. (1993) Reflex Sympathetic Dystrophy in Children: An Orthopedic Perspective. Orthopedics, 16, 773-779.

[5] Sherry, D.D., Wallace, C.A., Kelley, C., et al. (1999) Short- and Long-Term Outcomes of Children with Complex Regional Pain Syndrome Type I Treated with Exercise Therapy. Clinical Journal of Pain, 15, 218-223. http://dx.doi.org/10.1097/00002508-199909000-00009

[6] Goldsmith, D.P., Vivino, F.B., Eichenfield, A.H., et al. (1989) Nuclear Imaging and Clinical Features of Childhood Reflex Neurovascular Dystrophy: Comparison with Adults. Arthritis \& Rheumatology, 32, 480-485. http://dx.doi.org/10.1002/anr.1780320419 
[7] Laxer, R.M., Allen, R.C., Malleson, P.N., et al. (1985) Technetium 99m-Methylene Diphosphonate Bone Scans in Children with Reflex Neurovascular Dystrophy. Journal of Pediatrics, 106, 437-440.

http://dx.doi.org/10.1016/S0022-3476(85)80671-5

[8] Low, A. (2007) Pediatric Complex Regional Pain Syndrome. Journal of Pediatric Orthopedics, 27, 567-572. http://dx.doi.org/10.1097/BPO.0b013e318070cc4d

[9] Fitze, G. (2011) Complex Regional Pain Syndrome in Children. Unfallchirurg, 114, 411-416. http://dx.doi.org/10.1007/s00113-011-1964-3

[10] Cebrián, J. and Sánchez, P. (2009) Pediatric Complex Regional Pain Syndrome Affecting an Upper Limb: 7 Cases and a Brief Review of the Literature. Revista Española de Anestesiología y Reanimación, 56, 163-169. http://dx.doi.org/10.1016/S0034-9356(09)70358-5

[11] Logan, D.E., Williams, S.E., Carullo, V.P., Claar, R.L., Bruehl, S. and Berde, C.B. (2013) Children and Adolescents with Complex Regional Pain Syndrome: More Psychologically Distressed than Other Children in Pain? Pain Research \& Management, 18, 87-93.

[12] Borchers, A.T. and Gershwin, M.E. (2014) Complex Regional Pain Syndrome: A Comprehensive and Critical Review. Autoimmunity Reviews, 13, 242-265. http://dx.doi.org/10.1016/j.autrev.2013.10.006

[13] Matles, A.I. (1971) Reflex Sympathetic Dystrophy in a Child. A Case Report. Bulletin of the Hospital for Joint Diseases, 32, 193-197.

[14] Bétend, B., Lebacq, E., Kohler, R. and David, L. (1981) Metaphyseal Osteolysis. Unusual Aspect of Reflex Algodystrophy in Children. Archives Françaises de Pédiatrie, 38, 121-123.

[15] Wilder, R.T., Berde, C.B., Wolohan, M., Vieyra, M.A., Masek, B.J. and Micheli, L.J. (1992) Reflex Sympathetic Dystrophy in Children. Clinical Characteristics and Follow-Up of Seventy Patients. Journal of Bone and Joint Surgery, 74, 910-919.

[16] Zernikow, B., Dobe, M., Hirschfeld, G., Blankenburg, M., Reuther, M. and Maier, C. (2012) Please Don’t Hurt Me! A Plea against Invasive Procedures in Children and Adolescents with Complex Regional Pain Syndrome (CRPS). Schmerz, 26, 389-395.

[17] Greipp, M.E. (1990) Reflex Sympathetic Dystrophy Syndrome: A Retrospective Pain Study. Journal of Advanced Nursing, 15, 1452-1456. http://dx.doi.org/10.1111/j.1365-2648.1990.tb01788.x

[18] Greipp, M.E. (1988) Reflex Sympathetic Dystrophy Syndrome in Children. Pediatric Nursing, 14, 369-372.

[19] Tan, E.C., van de Sandt-Renkema, N., Krabbe, P.F.M., Aronson, D.C. and Severijnen, R.S.V.M. (2009) Quality of Life in Adults with Childhood-Onset of Complex Regional Pain Syndrome Type I. Injury, 40, 901-904. http://dx.doi.org/10.1016/j.injury.2009.01.134

[20] Linnman, C., Becerra, L., Lebel, A., Berde, C., Grant, P.E. and Borsook, D. (2013) Transient and Persistent Pain Induced Connectivity Alterations in Pediatric Complex Regional Pain Syndrome. PLoS ONE, 8, e57205. http://dx.doi.org/10.1371/journal.pone.0057205

[21] Lee, B.H., Scharff, L., Sethna, N.F., McCarthy, C.F., Scott-Sutherland, J., Shea, A.M., et al. (2002) Physical Therapy and Cognitive-Behavioral Treatment for Complex Regional Pain Syndromes. Journal of Pediatrics, 141, $135-140$. http://dx.doi.org/10.1067/mpd.2002.124380

[22] Matsui, M., Ito, M., Tomoda, A. and Miike, T. (2000) Complex Regional Pain Syndrome in Childhood: Report of Three Cases. Brain and Development, 22, 445-448. http://dx.doi.org/10.1016/S0387-7604(00)00174-1

[23] Häbler, H., Eschenfelder, S., Liu, X.G. and Jänig, W. (2000) Sympathetic-Sensory Coupling after L5 Spinal Nerve Lesion in the Rat and Its Relation to Changes in Dorsal Root Ganglion Blood Flow. Pain, 87, 335-345. http://dx.doi.org/10.1016/S0304-3959(00)00297-9

[24] Baron, R., Levine, J.D. and Fields, H.L. (1999) Causalgia and Reflex Sympathetic Dystrophy: Does the Sympathetic Nervous System Contribute to the Generation of Pain? Muscle \& Nerve, 22, 678-695. http://dx.doi.org/10.1002/(SICI)1097-4598(199906)22:6<678::AID-MUS4>3.0.CO;2-P

[25] Birklein, F., Weber, M., Ernst, M., Riedl, B., Neundörfer, B. and Handwerker, H.O. (2000) Experimental Tissue Acidosis Leads to Increased Pain in Complex Regional Pain Syndrome (CRPS). Pain, 87, 227-234. http://dx.doi.org/10.1016/S0304-3959(00)00286-4

[26] Forouzanfar, T., Koke, A.J., Van Kleef, M. and Weber, W.E.J. (2002) Treatment of Complex Regional Pain Syndrome Type I. European Journal of Pain, 6, 105-122. http://dx.doi.org/10.1053/eujp.2001.0304

[27] Verdugo, R.J. and Ochoa, J.L. (1998) Reversal of Hypoaesthesia by Nerve Block, or Placebo: A Psychologically Mediated Sign in Chronic Pseudoneuropathic Pain Patients. Journal of Neurology, Neurosurgery \& Psychiatry, 65, 196203. http://dx.doi.org/10.1136/jnnp.65.2.196

[28] Campero, M., Bostock, H., Baumann, T.K. and Ochoa, J.L. (2010) A Search for Activation of C Nociceptors by Sym- 
pathetic Fibers in Complex Regional Pain Syndrome. Clinical Neurophysiology, 121, 1072-1079. http://dx.doi.org/10.1016/j.clinph.2009.12.038

[29] Franklin, A. and Austin, T. (2013) The Use of a Continuous Brachial Plexus Catheter to Facilitate Inpatient Rehabilitation in a Pediatric Patient with Refractory Upper Extremity Complex Regional Pain Syndrome. Pain Practice, 13, 109113. http://dx.doi.org/10.1111/j.1533-2500.2012.00561.x

[30] Gibbons, J.J., Wilson, P.R., Lamer, T.J. and Elliott, B.A. (1992) Interscalene Blocks for Chronic Upper Extremity Pain. Clinical Journal of Pain, 8, 264-269. http://dx.doi.org/10.1097/00002508-199209000-00013

[31] Van Eijs, F., Stanton-Hicks, M., Van Zundert, J., Faber, C.G., Lubenow, T.R., Mekhail, N., et al. (2011) Complex Regional Pain Syndrome. Pain Practice, 11, 70-87. http://dx.doi.org/10.1111/j.1533-2500.2010.00388.x

[32] Capdevila, X., Bringuier, S. and Borgeat, A. (2009) Infectious Risk of Continuous Peripheral Nerve Blocks. Anesthesiology, 110, 182-188. http://dx.doi.org/10.1097/ALN.0b013e318190bd5b

[33] Meyer, M., Krane, E., Goldschneider, K. and Klein, N.J. (2012) Neurological Complications Associated with Epidural Analgesia in Children: A Report of 4 Cases of Ambiguous Etiologies. Anesthesia \& Analgesia, 115, 1365-1370. http://dx.doi.org/10.1213/ANE.0b013e31826918b6 
Scientific Research Publishing (SCIRP) is one of the largest Open Access journal publishers. It is currently publishing more than 200 open access, online, peer-reviewed journals covering a wide range of academic disciplines. SCIRP serves the worldwide academic communities and contributes to the progress and application of science with its publication.

Other selected journals from SCIRP are listed as below. Submit your manuscript to us via either submit@scirp.org or Online Submission Portal.
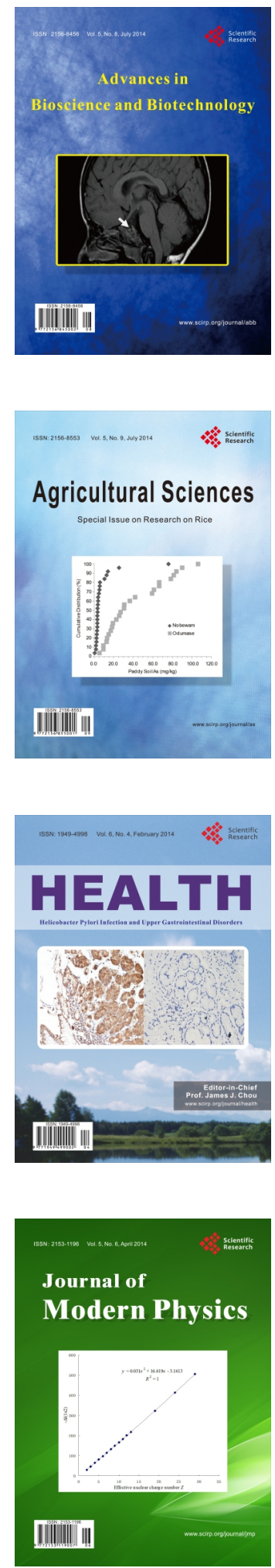
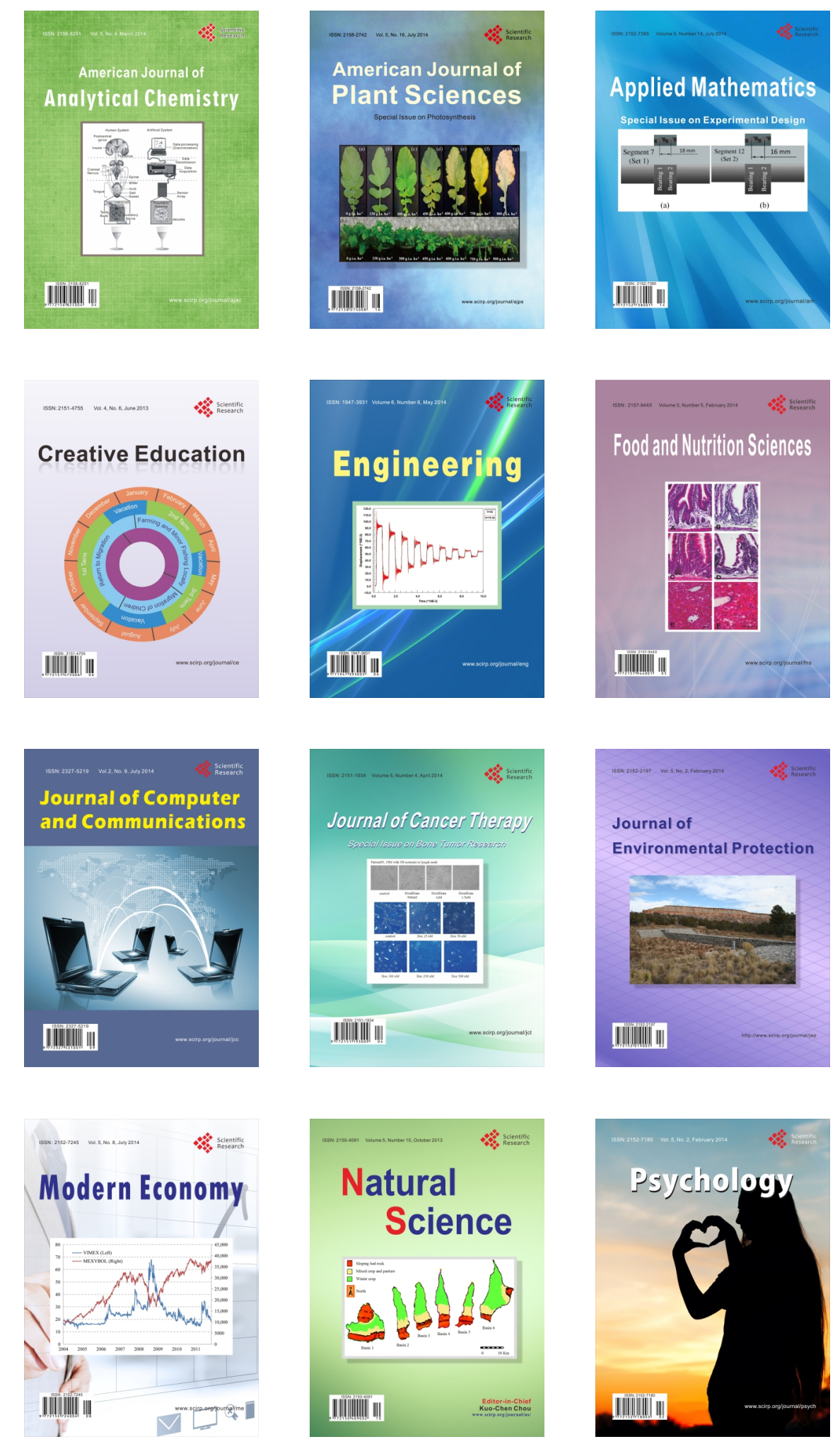